\title{
CUA-AUA International Fellows Program: Chicago 2019
}

\author{
Jesse Ory, MD \\ Department of Urology, Dalhousie University, Halifax, NS, Canada
}

Cite as: Can Urol Assoc J 2019;13(8):229. hitp://dx.doi.org/10.5489/cuaj.6124

was honored to be selected to take part in the Canadian Urological Association (CUA)-American Urological

Association (AUA) International Fellows Program and attend this year's AUA annual meeting. Along with four other Canadian residents, I traveled to Chicago for a combination of critical analysis of abstracts and plenaries, as well as to attend interviews for fellowships. The CUA-AUA program provides an excellent way to experience the AUA and get the most educational benefit from such a large meeting. Our goal under the CUA-AUA Program mandate is to broadly explore plenaries, podiums, and posters in oncology and bring the most interesting and thought-provoking abstracts for critical appraisal to our group.

One such abstract that caught my attention was a podium presentation by one of the authors of the SPARTAN trial on prostate-specific membrane antigen positron-emission tomography (PSMA PET) scans in patients with non-metastatic castrate-resistant prostate cancer (nmCRPC). ${ }^{1}$ The SPARTAN trial showed that in men with high-risk nmCRPC, who were non-metastatic and at most $\mathrm{N} 1$ by conventional imaging (PET and computed tomography [CT]), apalutamide improved median metastasis-free survival by 24.3 months. ${ }^{2}$ Over the past several years, PSMA PET has been shown to be able to detect prostate cancer metastasis and recurrence at significantly lower PSA levels than traditional imaging. The authors of this abstract thus proposed this question: how many SPARTAN-like men who are non-metastatic by conventional imaging would show disease with PSMA PET?

The authors collected data from six high-volume nuclear medicine centers, and selected 200 men who were as similar as possible in nature to men enrolled in the SPARTAN trial. Of these 200 men with at most N1 disease by conventional imaging, 98\% had a positive PSMA scan. Of key importance, $55 \%$ of these men had some form of extrapelvic distant metastatic disease; $39 \%$ had non-regional lymphadenopathy, $24 \%$ had bony metastases, and $6 \%$ had visceral metastases. Fifty-five percent had local recurrence and 54\% had regional nodal recurrence.

This trial is particularly interesting, as it identifies a patient subgroup that may benefit from PSMA scans. While these results do not negate the usefulness of apalutamide or

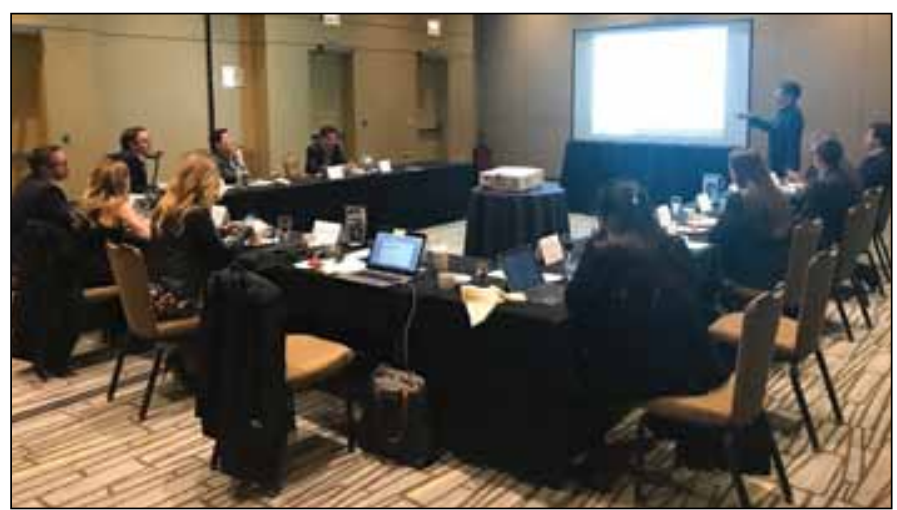

CUA-AUA fellows discussing high-impact abstracts following the AUA 2019 annual meeting.

enzalutamide in nmCRPC men, it does raise the possibility for future trials based on targeted therapy in these patients. With the ability to target the source of CRPC, could we potentially delay the need for systemic therapy even further? We may see an answer to this question at next year's AUA. One AUA attendee described a trial he has underway regarding targeted therapy in nmCRPC men based on PSMA targeted lesions.

The final feature of the CUA-AUA Fellows Program was gathering for a dinner and discussion of the highlights of the AUA. It was particularly interesting to take part in the discussion surrounding the critical appraisals that everyone brought to the table. This was an excellent opportunity to discuss new and exciting topics with experts in the field and helped to consolidate the knowledge I had gained from the conference. Comparing this year's AUA to my first AUA last year in San Francisco, it is easy to see how taking part in this program enriched my enjoyment of the meeting. I am grateful to the CUA, AUA, and industry partners for making this possible, and I strongly encourage any future residents and fellows to apply.

\section{References}

1. Hadischik $B$, Weber $M$, Irvani $A$, et al. Prostate-specific membrane antigen positron-emission tomography in high-risk non-metastatic castration-resistant prostate cancer SPARTAN-like patients negative by conventional imaging. Presented at American Urological Association Annual Meeting 2019, Chicago, IL: PD 17-09.

2. Smith MR, Saad F, Chowdhury $S$, et al. Apalutamide treatment and metastasis-free survival in prostate cancer. N Engl J Med 2018; 378:1408-18. https://doi.org/10.1056/NEJMoal715546

Correspondence: Dr. Jesse Ory, Department of Urology, Dalhousie University, Halifax, NS, Canada; jesse.ory@dal.ca 